\title{
The hidden information in patient-reported outcomes and clinician-assessed outcomes: multiple sclerosis as a proof of concept of a machine learning approach
}

\author{
Giampaolo Brichetto ${ }^{1,2}$ - Margherita Monti Bragadin ${ }^{1,2} \cdot$ Samuele Fiorini $^{3} \cdot$ Mario Alberto Battaglia $^{4}$. \\ Giovanna Konrad $^{2} \cdot$ Michela Ponzio $^{1} \cdot$ Ludovico Pedullà $^{1} \cdot$ Alessandro Verri $^{3} \cdot$ Annalisa Barla $^{3} \cdot$ Andrea Tacchino $^{1}$
}

Received: 8 March 2019 / Accepted: 28 September 2019/Published online: 28 October 2019

(C) The Author(s) 2019

\begin{abstract}
Machine learning (ML) applied to patient-reported (PROs) and clinical-assessed outcomes (CAOs) could favour a more predictive and personalized medicine. Our aim was to confirm the important role of applying ML to PROs and CAOs of people with relapsing-remitting (RR) and secondary progressive (SP) form of multiple sclerosis (MS), to promptly identifying information useful to predict disease progression. For our analysis, a dataset of 3398 evaluations from 810 persons with MS (PwMS) was adopted. Three steps were provided: course classification; extraction of the most relevant predictors at the next time point; prediction if the patient will experience the transition from RR to SP at the next time point. The Current Course Assignment (CCA) step correctly assigned the current MS course with an accuracy of about $86.0 \%$. The MS course at the next time point can be predicted using the predictors selected in CCA. PROs/CAOs Evolution Prediction (PEP) followed by Future Course Assignment (FCA) was able to foresee the course at the next time point with an accuracy of $82.6 \%$. Our results suggest that PROs and CAOs could help the clinician decision-making in their practice.
\end{abstract}

Keywords Multiple sclerosis $\cdot$ Disease course $\cdot$ Disease prediction $\cdot$ Patient-reported outcome $\cdot$ Machine learning

\section{Introduction}

Following the major revolution that is undergoing in medicine, the nature of healthcare is shifting from a disease centred to a patient-centred approach [1]. Beside emerging medical disciplines, such as genomic medicine, recently, participatory medicine is gaining increasing attention for diagnostic or therapeutic decision-making, consequently requiring new insights in patient involvement. Also, for multiple sclerosis (MS), innovative strategies to use traditional clinical measurements

Giampaolo Brichetto

giampaolo.brichetto@aism.it

1 Department of Research, Italian Multiple Sclerosis Foundation, Genoa, Italy

2 AISM Rehabilitation Center of Liguria, Genoa, Italy

3 Department of Informatics, Bioengineering, Robotics and System Engineering, University of Genoa, Genoa, Italy

4 Department of Life Science, University of Siena, Siena, Italy (i.e. patient-reported outcomes (PROs), clinician-assessed outcomes (CAOs)) and new computational tools (e.g. machine learning (ML)) could favour the shift from the current reactive medicine mode towards a personalized, predictive, preventive and participatory medicine [2]. In particular, the application of ML to PROs and CAOs could become the keystone [3] to better detect the rapid changes due to the pathology evolution and, consequently, to pave a timelier, low-cost and patient-centred way for people with MS (PwMS) management. Although, ML approaches [4] have proven to be able to extract meaningful information hidden in the data in a wide range of biomedical applications [5, 6], their role in analyzing PROs and CAOs of PwMS has still to be fully consolidated [7]. Several instrumental measures (e.g. MRI) offer established and well-known biomarkers of disease activity, especially for relapsing-remitting (RR) course of MS; those are currently less useful in detecting the transition from RR to the secondary progressive (SP) form [8]. Thus, ML applied to PROs and CAOs could be valuable to fill this gap or to improve MRI prediction power. Here, we applied previously developed machine learning algorithms [7] to a dataset 
Table 1 Personal, clinical, biometric, clinician assessed outcome (CAO) and patient reported outcomes (CAO) data acquired 4 months each from January-May 2014 to May-September 2017

Predictors contained in the vectors of the dataset

\begin{tabular}{|c|c|c|c|c|c|}
\hline & Code & Data type & No. of items & No. of subtotal scores & No. of total scores \\
\hline ABILHAND & ABILH & PRO & 23 & - & 1 \\
\hline Edinburgh Handedness Inventory & EHI & PRO & 10 & - & 1 \\
\hline Hospital Anxiety and Depression Scale & HADS & PRO & 14 & 2 & 1 \\
\hline Life Satisfaction Index & LSI & PRO & 11 & - & 1 \\
\hline Modified Fatigue Impact Scale & MFIS & PRO & 21 & 3 & 1 \\
\hline Overactive Bladder Questionnaire & OAB-q & PRO & 8 & - & 1 \\
\hline Functional Independence Measure & FIM & $\mathrm{CAO}$ & 19 & 6 & 1 \\
\hline Montreal Cognitive Assessment & $\mathrm{MoCA}$ & $\mathrm{CAO}$ & 11 & - & 2 \\
\hline Paced Auditory Serial Addition Task & PASAT & $\mathrm{CAO}$ & - & - & 1 \\
\hline Symbol Digit Modalities Test & SDMT & $\mathrm{CAO}$ & - & - & 1 \\
\hline Education (years) & EDU & Personal & - & - & 1 \\
\hline Relapses in the last 4 months (no.) & RELAPS & Clinical & - & - & 1 \\
\hline Height $(\mathrm{cm})$ & $\mathrm{H}$ & Biometric & - & - & 1 \\
\hline Weight $(\mathrm{kg})$ & $\mathrm{W}$ & Biometric & - & - & 1 \\
\hline \multicolumn{6}{|c|}{ Predictors selected in disease course classification and prediction } \\
\hline ABILH (item 12) & \multicolumn{5}{|c|}{ Hammering a nail } \\
\hline ABILH (TOT) & \multicolumn{5}{|c|}{ ABILH total score } \\
\hline HADS (item 7) & \multicolumn{5}{|c|}{ I can sit at ease and feel relaxed. } \\
\hline HADS (SUB1) & \multicolumn{5}{|c|}{ HADS subtotal measuring anxiety } \\
\hline HADS (SUB2) & \multicolumn{5}{|c|}{ HADS subtotal measuring depression } \\
\hline HADS (TOT) & \multicolumn{5}{|c|}{ HADS total score } \\
\hline LSI (TOT) & \multicolumn{5}{|c|}{ LSI total score } \\
\hline MFIS (item 2) & \multicolumn{5}{|c|}{ I have had difficulty paying attention for long periods of time. } \\
\hline MFIS (SUB1) & \multicolumn{5}{|c|}{ MFIS subtotal measuring cognitive status } \\
\hline MFIS (SUB2) & \multicolumn{5}{|c|}{ MFIS subtotal measuring physical status } \\
\hline MFIS (SUB3) & \multicolumn{5}{|c|}{ MFIS subtotal measuring psychosocial status } \\
\hline MFIS (TOT) & \multicolumn{5}{|c|}{ MFIS total score } \\
\hline OAB-q (item 1) & \multicolumn{5}{|c|}{ Frequent urination during the daytime hours } \\
\hline OAB-q (item 4) & \multicolumn{5}{|c|}{ Accidental loss of small amounts of urine } \\
\hline OAB-q (TOT) & \multicolumn{5}{|c|}{ OAB-q total score } \\
\hline FIM (item 10) & \multicolumn{5}{|c|}{ How much assistance is required for toilet transfer? } \\
\hline FIM (item 11) & \multicolumn{5}{|c|}{ How much assistance is required for shower transfer? } \\
\hline FIM (item 12) & \multicolumn{5}{|c|}{ How much assistance is required for locomotion (ambulatory)? } \\
\hline FIM (item 14) & \multicolumn{5}{|c|}{ How much assistance is required for locomotion (wheelchair)? } \\
\hline FIM (SUB3) & \multicolumn{5}{|c|}{ FIM subtotal measuring sphincter control } \\
\hline FIM (SUB4) & \multicolumn{5}{|c|}{ FIM subtotal measuring personal care } \\
\hline FIM (SUB5) & FIM subto & suring locom & & & \\
\hline FIM (SUB6) & FIM subto & Isuring mobil & & & \\
\hline FIM (TOT) & FIM total s & & & & \\
\hline MoCA (item 1) & Visuocons & al test & & & \\
\hline MoCA (item 9) & Memory te & & & & \\
\hline MoCA (TOT1) & MOCA to & & & & \\
\hline MoCA (TOT2) & MOCA to & e corrected fc & of education & & \\
\hline PASAT & Score & & & & \\
\hline SDMT & Score & & & & \\
\hline EDU & Years of $\mathrm{fo}$ & ducation & & & \\
\hline $\mathrm{H}$ & Height ( $\mathrm{cn}$ & & & & \\
\hline $\mathrm{W}$ & Weight $(\mathrm{kg}$ & & & & \\
\hline
\end{tabular}

(PROMOPRO-MS database) populated of PROs and CAOs exclusively from RR and SP PwMS, by confirming the decisive role that ML approaches could play in the future in timely identifying information useful to predict the progression from RR to SP course and consequently in supporting pharmacological and rehabilitative therapeutic decision-making in order to prevent this transition.

\section{Materials and methods}

Patients followed as outpatients or at-home by Italian Multiple Sclerosis Society (AISM) Rehabilitation Centres of Genoa,
Padua and Vicenza, have been progressively enrolled in the study [9] without any inclusion/exclusion criteria unless MS diagnosis. The study was approved by the local ethics committee (Comitato Etico Aziendale A.O. Universitaria "San Martino" Genova). A written informed consent was obtained from all subjects prior to study entry.

Personal (i.e. years of education), clinical (i.e. number of relapses in the last 4 months) and biometric (i.e. height and weight) data, PROs and CAOs related to the most relevant domains for MS (i.e. mobility, fatigue, cognitive performances, emotional status, bladder continence, quality of life), were acquired each 4 months from January-May 2014 to May-September 2017 for a maximum of 11 evaluations for 
each participant. Each sample of the dataset is represented by a vector containing 143 predictors (i.e. single question, subtotal scores and total scores) (Table 1) and adopted to finally refine previously developed ML algorithms [7].

We proceeded through a three-step strategy based on supervised ML algorithms in order to develop a MS evolution temporal model (i.e. from RR to SP): Current Course Assignment (CCA), PROs/CAOs Evolution Prediction (PEP) and Future Course Assignment (FCA). In particular, CCA solves a binary classification problem: each predictor vector, independently of evaluations time course, is associated with the most probable MS form (RR or SP). PEP provides an updated historical representation: for each patient, the most probable predictor vector at the next time point is predicted. FCA model is just the function composition of FCA and PEP. Therefore, it allows foreseeing if the patient at the next time point will be RR or SP, by allowing inferring if he/she will experience the transition.

\section{Results}

A total of 3398 evaluations from 810 PwMS represented the dataset adopted for our analysis. Among those, 1451 were RR PwMS at the evaluation time and 1947 SP PwMS. The CCA step correctly assigned the current MS course with an accuracy of about $86.0 \%$. This was obtained through a reduced number of predictors from the considered PROs and CAOs and from biometric and personal data (Table 1). Specifically, selfand physician-reported information of the physical domain (upper limb abilities, fatigue, personal care and locomotion) and few self-reported information of present and past mood and quality of life, as well as clinically assessed performances in cognitive tests (memory, calculation, information processing), were necessary.

Although important, the most interesting result was obtained from the second and third steps of the analysis. By using the predictors selected in CCA, PEP followed by FCA was able to foresee the course at the next time point with an accuracy of $82.6 \%$.

The results suggest that the disease course prediction based on PROs and CAOs is feasible in MS. However, although a reduced number of predictors is selected with respect to those originally considered, disease course prediction at the next time point requires to acquire all 143 predictors.

\section{Conclusion}

Here, we presented a data analysis pipeline based on ML methods that could address MS diagnosis and prognosis issues. Our results show that PROs and CAOs could be used to build accurate models of MS disease course prediction. PROs and CAOs, alone or integrated with other indexes such as MRI outcomes and biomarkers, could help the decision-making process of clinicians in their daily practice. The main limitation of the current prediction algorithm is the considerable amount of data needed. Future developments of the algorithm that will integrate also data on therapies followed by the patients already present in the current database and eventually MRI outcomes will take into account the necessity to optimize and minimize the necessary data. In conclusion, the possibility to better define the clinical complexity levels of the patient and to have sufficient and adequate predictive criteria for MS evolution could be an instrument for the construction of a more fruitful therapeutic pact between clinician and patient based on better perspective knowledge, increased disease consciousness and engagement in pharmacological and rehabilitative treatments, and physical activity, to date, is considered the main way to delay MS progression and to improve quality of life [10].

Acknowledgements The authors thank Maria Madera and Giulia Bignone for their fundamental role in enrollment and scheduling of the participants to the project.

Funding information This study has been supported by Italian MS Foundation (FISM/2013/S3 - PROMOPRO-MS and FISM/2015/R3 DETECT-MS).

\section{Compliance with ethical standards}

The study was approved by the local ethics committee (Comitato Etico Aziendale A.O. Universitaria "San Martino" Genova). A written informed consent was obtained from all subjects prior to study entry.

Open Access This article is distributed under the terms of the Creative Commons Attribution 4.0 International License (http:// creativecommons.org/licenses/by/4.0/), which permits unrestricted use, distribution, and reproduction in any medium, provided you give appropriate credit to the original author(s) and the source, provide a link to the Creative Commons license, and indicate if changes were made.

\section{References}

1. Miller A, Avidan N, Tzunz-Henig N, Glass-Marmor L, Lejbkowicz I, Pinter RY, Paperna T (2008) Translation towards personalized medicine in multiple sclerosis. J Neurol Sci 274(1-2):68-75

2. Giovannoni $G$ (2017) Personalized medicine in multiple sclerosis. Neurodegener Dis Manag 7(6s):13-17

3. Lejbkowicz I, Caspi O, Miller A (2012) Participatory medicine and patient empowerment towards personalized healthcare in multiple sclerosis. Expert Rev Neurother 12(3):343-352

4. Bishop CM (2012) Model-based machine learning. Philos Trans A Math Phys Eng Sci 371(1984):20120222

5. Speicher NK, Pfeifer N (2015) Integrating different data types by regularized unsupervised multiple kernel learning with application to cancer subtype discovery. Bioinformatics 31(12):i268-i275 
6. Di Camillo B, Sanavia T, Martini M et al (2012) Effect of size and heterogeneity of samples on biomarker discovery: synthetic and real data assessment. PLoS One 7(3):e32200

7. Fiorini S, Verri A, Barla A, et al. Temporal prediction of multiple sclerosis evolution from patient-centered outcomes. In: Machine learning for healthcare conference (PMLR), Boston, Massachusetts, 18-19 August 2017, volume 68, pp.112-125. http://proceedings.mlr.press/v68/fiorini17a/fiorini17a.pdf (accessed 23 July 2019)

8. Filippi M, Agosta F (2010) Imaging biomarkers in multiple sclerosis. J Magn Reson Imaging 31(4):770-788
9. Bebo BF Jr, Fox RJ, Lee K et al (2018) Landscape of MS patient cohorts and registries: recommendations for maximizing impact. Mult Scler 24(5):579-586

10. Platform EMS. Recommendation on rehabilitation services for persons with multiple sclerosis in Europe, www.eurims.org/files/202/ Guidelines-and-Recommendations/256/Recommendations-onMS-Rehabiltation-RIMS-EMSP-2012.pdf (2018, accessed 19 July 2018).

Publisher's note Springer Nature remains neutral with regard to jurisdictional claims in published maps and institutional affiliations. 